Mots. Les langages du politique

De la racine à l'extrémisme : discours des radicalités politiques et sociales

Maria Candea et Laélia Véron, Le français est à nous! Petit manuel d'émancipation linguistique

Paris, La Découverte, 2019

Hadrien Clouet

\title{
OpenEdition
}

Journals

Édition électronique

URL : https://journals.openedition.org/mots/26943

DOI : $10.4000 /$ mots. 26943

ISBN : 979-10-362-0250-6

ISSN : 1960-6001

Éditeur

ENS Éditions

Édition imprimée

Date de publication : 10 septembre 2020

Pagination : 123-129

ISBN : 979-10-362-0249-0

ISSN : 0243-6450

Référence électronique

Hadrien Clouet, « Maria Candea et Laélia Véron, Le français est à nous ! Petit manuel d'émancipation linguistique », Mots. Les langages du politique [En ligne], 123 | 2020, mis en ligne le 01 janvier 2021, consulté le 03 janvier 2023. URL : http://journals.openedition.org/mots/26943 ; DOI : https://doi.org/ $10.4000 /$ mots. 26943

Ce document a été généré automatiquement le 3 janvier 2023

Tous droits réservés 


\section{Maria Candea et Laélia Véron, Le français est à nous! Petit manuel d'émancipation linguistique}

Paris, La Découverte, 2019

\section{Hadrien Clouet}

\section{RÉFÉRENCE}

Maria Candea et Laélia Véron, Le français est à nous ! Petit manuel d'émancipation linguistique. Paris, La Découverte, 2019

1 La langue française est-elle menacée par ses évolutions? Oui, se lamentent d'illustres « hommes de lettres» depuis 1000 ans. Non, ripostent les deux autrices, montrant de façon implacable les luttes incessantes qui traversent le français dès ses premières codifications. Difficilement classable, l'ouvrage est à la fois une analyse sociolinguistique, une revue de littérature et un plaidoyer pour une action publique moderniste en matière de langue française.

2 Il est divisé en trois grandes parties, qui correspondent à autant de thèses.

3 La première partie défend la thèse selon laquelle les langues sont des produits vivants et malléables. Le chapitre I revient sur leur construction. Les autrices montrent la difficulté de toute opération de définition d'une langue qui « se fonde sur l'observation d'un minuscule échantillon » (p. 17) et souffre de l'absence de tout enregistrement oral avant la fin $\mathrm{du} \mathrm{XIX}^{\mathrm{e}}$ siècle. Aussi la description d'une langue est-elle tributaire des milieux sociaux étudiés et aussi des bornes historiques retenues pour reconstituer sa genèse a posteriori - les Serments de Strasbourg sont « enseigné[s] comme le premier texte en français» (p.19), car ils furent prononcés dans une ville actuellement préfecture du Grand Est, mais demeurent largement incompréhensibles des locuteurs contemporains. La langue est donc une invention politique rétrospective. Elle est reproduite et renouvelée au quotidien par des organisations, qui luttent pour imposer 
leur propre répertoire de mots et la graphie de chacun: dictionnaires concurrents, Commission d'enrichissement de la langue française, Académie française, journaux, logiciels automatiques de correction, blogs, plateformes collaboratives et mouvements sociaux... Ainsi, «nos usages linguistiques sont trop divers et trop mouvants pour qu'ils puissent être répertoriés dans une liste de formes reconnues comme correctes, fût-elle de très grande taille » (p. 33).

4 Si la langue est un espace mouvant et contesté, qu'est-ce alors qu'une «faute »? Les autrices nous montrent dans le second chapitre qu'elle dépend du rapport de force entre les locuteurs et les institutions linguistiques: «lorsque nous avons assez de capital culturel pour nous sentir légitimes, nous n'hésitons pas à renégocier les règles, à déplacer les frontières de ce qui est beau et/ou acceptable. C'est d'ailleurs ce que font écrivains et écrivaines" (p.45). Ces luttes incessantes conduisent même à un éclatement de la langue française et à la dispersion territoriale des grammaires ordinaires, puisque "le français standard évolue d'une région à une autre [sans] consensus sur les critères à mobiliser pour décider de ce qui en relève ou non » (p. 54). Les autrices privilégient la perspective d'un continuum entre registres (différenciés) de langue, plutôt que d'une rupture entre niveaux (hiérarchisés) de langue. Remontant à la Grammaire des fautes d'Henri Frei (1920), elles nous montrent la fréquence des situations dans lesquelles "c'est la variante considérée comme erronée qui a fini par s'imposer comme correcte» (p.55). La faute, ou plus précisément et moins normativement l'erreur, est donc un terme qui n'est pas ou plus accepté par le grand nombre.

5 Le troisième chapitre s'intéresse aux discours catastrophistes sur le "danger » de disparition du français. $\mathrm{Si}$ « des centaines de langues humaines ont disparu dans les décennies précédentes» (p.63), la langue française semble bien préservée - elle est plus homogène en 2019 que dans toute son histoire. Les discours appelant à défendre la langue française contre l'extinction sont légion depuis le pamphlet Champ fleury de Geoffroy Tory au $\mathrm{XVI}^{\mathrm{e}}$ siècle. Leur constance ne doit pas dissimuler la variation de la menace identifiée : les inventeurs de mots en 1529, l'administration en 1930, la jeunesse en 1990, et particulièrement la jeunesse immigrée à partir des années 2000. Le goût du français apparaît comme le dégoût de certaines populations. Pour le dire avec Gramsci, lorsqu' " affleure, d'une manière ou d'une autre, la question de la langue, cela veut dire qu'une série d'autres problèmes sont en train de s'imposer » (Gramsci, 1996).

6 Cependant, la langue n'est pas qu'un espace de lutte interne pour déterminer un périmètre de mots et une correction grammaticale, ni un simple prétexte. Elle constitue un instrument importé dans d'autres conflits sociaux. La partie II du livre les décline : rapports de pouvoir, de genre, coloniaux. Or, comme la critique de l'économie politique conteste la neutralité de la monnaie, le chapitre IV démystifie la prétention de la langue à représenter un voile sur les échanges. La performativité des échanges acte des hiérarchies sociales, réduit la dignité d'individus ou leur attribue une identité refusée, constituant une «violence symbolique [qui] permet de prendre le pouvoir sur l'interlocuteur ou l'interlocutrice» (p. 92). Les autrices puisent chez Pierre Bourdieu, Frantz Fanon et Marina Yaguello plusieurs clés de leur démonstration, afin de souligner la force symbolique des mots, indépendante des intentions des locuteurs.

7 Le chapitre $\mathrm{V}$ se concentre sur une lutte linguistique : la féminisation des mots. Il rappelle la mobilisation des "grammairiens interventionnistes » (expression d'Éliane Viennot, p.106) au $\mathrm{XVII}^{\mathrm{e}}$ siècle, soucieux d'instaurer le masculin générique (appelé 
"genre noble " jusqu'au $\mathrm{xx}^{\mathrm{e}}$ siècle, p. 108), balayant les règles antérieures de l'accord de proximité. Toutefois, des résistances langagières ont su en limiter l'emprise, dans les dialogues quotidiens, dans certains journaux ou même dans l'administration, l'accord de proximité étant accepté «dans les épreuves de français pour les concours de la fonction publique jusqu'en 1976»(p.110). Loin de représenter une lubie récente, l'écriture inclusive constitue plutôt un souci de rétablir un équilibre genré, effacé il y a peu de la langue française, tout en cessant d'asséner à «un groupe composé exclusivement de filles qu'on s'adresse à elles au masculin pluriel dès qu'un garçon les rejoint» (p.119). Le chapitre se clôt sur une explication très pédagogique du contenu de « la communication inclusive, qui vise à une représentation égale des femmes et des hommes dans la langue» (p. 117). Loin de réduire la question à la seule forme écrite, et sa solution au seul point médian, les autrices déploient le répertoire inclusif dans son entièreté : accord des noms de métiers, doublets, féminisation par points médians, parenthèses ou tirets... Ce faisant, elles rappellent qu'une partie de la communication inclusive a déjà été conquise depuis les années 1970.

Le chapitre VI aborde l'enjeu colonial de la langue. Pour cela, il rappelle que l'élévation du niveau de connaissances scolaires n'a jamais été un objectif des politiques coloniales. La diffusion du français était très inégalitaire et concentrée chez les élites : un français colonial, construit de toutes pièces, était réservé aux masses colonisées. "L'enseignement d'une langue française au rabais » s'appuie essentiellement sur le volet «oral, pour favoriser les échanges pratiques» (p.128). L'encadré sur la construction du « français petit nègre » par l'armée coloniale est éclairant à cet égard. La «mission civilisatrice » est plutôt une segmentation de l'écrit, soulignant que le "vol d'histoire» (Goody, 2015) repose aussi sur l'expropriation des instruments de « raison graphique » (Goody, 1979). Les autrices s'arrêtent sur les déclinaisons locales de cette orientation, en Algérie, en Afrique-Occidentale française et en NouvelleCalédonie, pour conclure paradoxalement à une expansion du français au moment de la décolonisation.

La place du français hors de France est prolongée par le chapitre VII, qui se penche sur l'Organisation internationale de la francophonie (OIF). Les autrices mettent quatre contradictions au jour: l'OIF arbore une vocation linguistique alors qu'elle se rapproche du modèle d'un espace économique et commercial; l'OIF revendique la coopération de pays francophones alors qu'elle compte un certain nombre de membres non ou peu francophones; la coopération linguistique francophone est souvent réduite à l'OIF malgré la densité et la vitalité de la communauté mondiale francophone ( « citons par exemple le premier Parlement des écrivaines francophones, qui s'est tenu à Orléans en 2018 », p. 154); la France continue d'exercer un rôle prééminent alors qu'elle regroupe une proportion de plus en plus réduite des locuteurs francophones.

Finalement, la troisième partie propose de parcourir la genèse de la langue française, à travers ses controverses, de la Renaissance à l'époque actuelle. Le chapitre VIII se penche ainsi sur la notion de "génie » de la langue française, rapidement déconstruit et réduit au rang de discours idéologique et apologétique, au service d'ambitions géopolitiques et chauvines. D'abord outil de distinction sociale, le français est ensuite naturalisé, dans la foulée de Rivarol, soucieux de le présenter comme «la seule langue dont l'ordre des mots suivrait le raisonnement naturel» (p.163). Les autrices démontrent que les louanges adressées au français par ses partisans suprématistes reposent sur une disqualification systématique des langues concurrentes: l'italien 
(xVI ${ }^{\mathrm{e}}-\mathrm{XVII}{ }^{\mathrm{e}}$ siècle), l'espagnol $(\mathrm{xVII})$, l'anglais $(\mathrm{xVIII})$. Une approche rigoureuse des questions linguistiques implique dès lors «d'adopter un regard apaisé, moins méprisant par rapport aux autres langues » (p. 172).

Le chapitre IX revient sur la politique révolutionnaire vis-à-vis des langues, entre 1789 et 1794 : unification nationale, invention de néologismes, décret sur le tutoiement obligatoire, substitution scolaire du français au grec et au latin... Les autrices insistent sur les limites de l'action révolutionnaire en ce domaine, soit par incomplétude (plusieurs organismes publics périclitent rapidement faute de moyens), soit par coercition (l'hostilité aux parlers régionaux se mue en tentative d'écrasement, qui provoque des résistances vives). Elles en concluent que «la langue a été un terrain d'expérimentations politiques surtout pour l'élite révolutionnaire » (p. 184).

Le chapitre $\mathrm{X}$ analyse les contradictions de la scolarisation de masse engagée par la monarchie de Juillet et poursuivie sous la III ${ }^{e}$ République. Son aspiration à codifier une orthographe d'État a largement débordé le cadre scolaire pour s'étendre à toute la vie sociale. En témoigne l'injonction policière faite aux commerçants de corriger leurs affichages. Ce durcissement des codes s'est accompagné, paradoxalement, d'un blocage de toute réforme orthographique, «de peur de faire échouer l'entreprise d'une envergure sans précédent d'enseignement généralisé " (p.191). Les interrogations pédagogiques remplacent alors les interrogations sur la construction de la langue ellemême.

13 Le chapitre XI effectue un grand bond vers la révolution numérique pour interroger la digitalisation du langage. Là encore, plusieurs discours conservateurs sont battus en brèche. D'abord, la propagation de l'accès à internet et des téléphones mobiles a élargi le champ de l'écrit comme jamais auparavant dans l'histoire humaine. Ensuite, une partie de la gêne éprouvée par certaines institutions linguistiques provient de «l'apparition de nouvelles formes de communication écrite qui ne s'inscrivaient dans aucune tradition établie et ont dû inventer, en un temps très court, leurs codes, règles de politesse, conventions" (p.206). Le numérique n'a pas déstabilisé la langue française, mais démultiplié ses modes d'appropriation. Loin de révolutionner intégralement les formats graphiques, il rappelle l'usage fort ancien des abréviations par les copistes. Rassurantes, les autrices convoquent un certain nombre d'études qui témoignent de l'absence d'effet de la phonographie (rédaction proche de la prononciation, comme «tuvoi») sur le niveau scolaire en orthographe - au contraire, «cet assouplissement des normes nous a permis, et c'est nouveau, de prendre des positions plus personnelles sur la question des graphies» (p. 209).

Dans leur conclusion, les autrices développent trois idées fortes qui synthétisent les objectifs du livre. D'abord, elles contestent le fait que les opposants à l'évolution de la langue soient attachés à cette dernière. Elles jugent qu'ils "détestent la langue française. Celle-ci, telle qu'elle est parlée, écrite, interrogée, enrichie, malaxée, bref, telle qu'elle existe, dans son immense diversité, ne les intéresse pas » (p. 219). Ensuite, elles plaident en faveur de débats ouverts et publics autour des évolutions attendues de la langue. En dernier lieu, elles incriminent les discours catastrophistes en matière linguistique (sur la réintroduction du prédicat, la suppression de «mademoiselle »...), qui verrouillent le sujet et le laissent aux seules mains de « celles et ceux qui ne veulent surtout pas que leur statut (contestable) "d'experts" soit remis en cause » (p. 224).

La structure de l'ouvrage est très pédagogique, car chaque chapitre s'ouvre sur une synthèse («on pense à tort que...» / «on ne sait pas que...») et comporte une 
bibliographie réduite au strict minimum. Il se clôt sur un glossaire final. Selon la préoccupation des lecteurs, le plan adopté sera soit avantageux (il permet de circuler librement entre les chapitres, de passer d'une thématique à une autre) soit déroutant (pour les adeptes des récits chronologiques, déboussolés par les allers-retours). Il ne faut donc pas l'appréhender comme une histoire critique de la langue française, mais bien comme une critique de l'histoire de la langue française, qui permet aux autrices de défendre une conception descriptive plutôt que normative de la sociolinguistique.

Les lecteurs pourront aussi s'approprier l'ouvrage comme la photographie sociale d'une controverse montante. Ils le rapprocheront utilement des travaux de sociologie historique pour comprendre la montée en puissance contemporaine de ces problématiques, notamment de ceux qui soulignent comment «la hantise du déclin international » et la "tertiarisation de l'économie et de l'emploi » ont pu accoucher d'une coalition élitiste, composée d'élites gouvernementales en charge du rayonnement national et d'une petite bourgeoisie en ascension soucieuse d'adopter les codes supérieurs, qui communient dans la défense acharnée d'une langue française intangible (Dubois, 2003).

Un autre point fort du livre consiste à traduire les enjeux linguistiques sur le plan des ressources matérielles. Il montre bien que la langue est un espace d'investissements, symboliques, économiques, culturels et sociaux, inégalement convertibles selon l'époque et les institutions dominantes, qui octroient à certains le privilège de façonner la langue d'une manière plus légitime. La langue est le produit de luttes inégalitaires.

L'ampleur de l'argumentation suscitera forcément des regrets de détails. Ainsi, les institutions étudiées demeurent nettement circonscrites aux organismes publics ou associatifs nationaux (à l'exception, notable, de l'OIF). Le rôle des sociétés commerciales dans les recompositions linguistiques n'est pas abordé de front (Truchot, 2008). De même, le rôle pacificateur de la traduction simultanée et le rôle intégrateur du plurilinguisme dans les espaces supranationaux ne sont pas évoqués, alors qu'ils modifient des milieux comme le syndicalisme (Wagner, 2005).

Le pluralisme des acteurs permet de comprendre la complexité des évolutions linguistiques, leur caractère inachevé et conflictuel. Cependant, ce pluralisme aurait aussi pu être historicisé en tant que configuration particulière : le contrepoint de l'encadrement dictatorial des langues n'est pas mentionné, alors qu'il aurait sans aucun doute mis en valeur les processus expliqués (Klemperer, 2003 ; Matard-Bonucci, 2018).

L'absence des autres constructions linguistiques nationales empêche quelque peu de déterminer à quel point la langue française est le produit de logiques idiosyncratiques. Ainsi, l'encadrement étroit de la langue par les révolutionnaires français apparaît en opposition totale à leurs successeurs soviétiques, bien plus libéraux (Jakubinskij, 2012), tandis que l'objet des débats linguistiques paraît très différent de ceux qui déchirent, par exemple, l'espace germanique, travaillé depuis longtemps par des conférences orthographiques (Polenz, 2009). La fabrication intégrale de langues, comme l'espéranto, aurait aussi pu fournir des éléments précieux de qualification et de dénaturalisation des spécificités françaises.

21 Mais ces quelques remarques ne remettent aucunement en cause l'intérêt de l'ouvrage, à la fois pour comprendre les évolutions du français et pour opérer un retour réflexif sur notre propre position sociale de locuteur. À chaque fois qu'ils ouvrent la bouche, lecteurs et lectrices se rendront compte qu'ils font de la politique. 


\section{BIBLIOGRAPHIE}

DUBoIs Vincent, 2003, «Comment la langue devient une affaire d'État : la défense de la langue française au milieu des années 1960 », dans J. Lagroye éd., La politisation, Paris, Belin, p. 461-474. GOODY Jack, 2015, Le vol de l'histoire : comment l'Europe a imposé le récit de son passé au reste du monde, F. Durand-Bogaert trad., Paris, Gallimard. GOODY Jack, 1979, La raison graphique : la domestication de la pensée sauvage, J. Bazin et A. Bensa trad., Paris, Minuit.

GRAMSCI Antonio, 1996, Cahiers de prison. I, Cahiers 1, 2, 3, 4, 5, R. Paris éd., M. Aymard et F. Bouillot trad., Paris, Gallimard.

JAKUBINSKIJ Lev, 2012, Une linguistique de la parole (URSS, années 1920-1930), I. Ivanova éd., I. Ivanova et P. Sériot trad., Limoges, Lambert-Lucas, Lausanne, CRECLECO.

KLEMPERER Victor, 2003, LTI, la langue du III Reich : carnets d'un philologue, É. Guillot trad., Paris, Pocket.

MATARD-BONUCCI Marie-Anne, 2018, Totalitarisme fasciste, Paris, CNRS Éditions.

POLENZ Peter von, 2009, Geschichte der deutschen Sprache, Berlin, De Gruyter.

TRUснот Claude, 2008, Europe : l'enjeu linguistique, Paris, La Documentation française.

WAGNER Anne-Catherine, 2005, Vers une Europe syndicale : une enquête sur la Confédération européenne des syndicats, Bellecombe-en-Bauges, Éditions du Croquant.

\section{AUTEURS}

\section{HADRIEN CLOUET}

Centre de sociologie des organisations (CNRS-Sciences Po) 